\title{
X-ray properties of NGC 300
}

\section{Global properties of X-ray point sources and their optical counterparts}

\author{
S. Carpano ${ }^{1}$, J. Wilms $^{2}$, M. Schirmer ${ }^{3,4}$, and E. Kendziorra ${ }^{1}$ \\ 1 Institut für Astronomie und Astrophysik, Abteilung Astronomie, Universität Tübingen, Sand 1, 72076 Tübingen, Germany \\ e-mail: carpano@astro.uni-tuebingen.de \\ 2 Department of Physics, University of Warwick, Coventry, CV4 7AL, UK \\ 3 Institut für Astrophysik und Extraterrestrische Forschung, Universität Bonn, Auf dem Hügel 71, 53121 Bonn, Germany \\ ${ }^{4}$ Isaac Newton Group of Telescopes, 38700 Santa Cruz de La Palma, Spain
}

Received 12 October 2004 / Accepted 14 July 2005

\section{ABSTRACT}

We present X-ray properties of NGC 300 point sources, extracted from $66 \mathrm{ks}$ of XMM-Newton data taken in 2000 December and 2001 January. A total of 163 sources were detected in the energy range of $0.3-6 \mathrm{keV}$. We report on the global properties of the sources detected inside the $D_{25}$ optical disk, such as the hardness ratio and X-ray fluxes, and on the properties of their optical counterparts found in $B, V$, and $R$ images from the $2.2 \mathrm{~m} \mathrm{MPG/ESO}$ telescope. Furthermore, we cross-correlate the X-ray sources with SIMBAD, the USNO-A2.0 catalog, and radio catalogues.

Key words. galaxies: individual: NGC 300 - X-rays: galaxies

\section{Introduction}

Studies of the X-ray population of spiral galaxies other than our Galaxy are of importance especially for the understanding of the formation of X-ray binaries and other X-ray emitting sources. NGC 300 is a member of the Sculptor galaxy group. Due to its small distance $(\sim 2.02 \mathrm{Mpc}$; Freedman et al. 2001), the SA(s)d dwarf galaxy NGC 300 is an ideal target for the study of the entire X-ray population of a typical normal quiescent spiral galaxy. The major axes of the $D_{25}$ optical disk are $13.3 \mathrm{kpc}$ and $9.4 \mathrm{kpc}\left(22^{\prime} \times 15^{\prime}\right.$; de Vaucouleurs et al. 1991). These studies are even more simplified by the galaxy's almost face-on orientation and its low Galactic column density $\left(N_{\mathrm{H}}=3.6 \times 10^{20} \mathrm{~cm}^{-2}\right.$; Dickey \& Lockman 1990).

The first X-ray population study of NGC 300 was performed between 1991 and 1997 with a total of five ROSAT pointings (Read \& Pietsch 2001). The total exposure time of these data was $46 \mathrm{ks}$ in the ROSAT Position Sensitive Proportional Counter and $40 \mathrm{ks}$ in the ROSAT High Resolution Imager, all with a nominal pointing position of $\alpha_{\mathrm{J} 2000.0}=$ $00^{\mathrm{h}} 54^{\mathrm{m}} 52^{\mathrm{s}} .0$ and $\delta_{\mathrm{J} 2000.0}=-37^{\circ} 41^{\prime} 24^{\prime \prime} 0$. In these observations, a total of 29 sources was discovered within the $D_{25}$ disk, the brightest being a black hole candidate with

* Tables 1 and 2 are also available in electronic form at the CDS via anonymous ftp to cdsarc.u-strasbg.fr (130.79.128.5) or via http://cdsweb.u-strasbg.fr/cgi-bin/qcat? J/A+A/443/103
$L_{\mathrm{X}}=2.2 \times 10^{38} \mathrm{erg} \mathrm{s}^{-1}$ in the $0.1-2.4 \mathrm{keV}$ band. Read \& Pietsch (2001) also identified a highly variable supersoft source and other bright sources coincident with known supernova remnants (SNRs) and H II regions. The luminosity of the residual $\mathrm{X}$-ray emission, probably due to unresolved sources and genuine diffuse gas, has been estimated to $L_{\mathrm{X}}=1.2 \times 10^{38} \mathrm{erg} \mathrm{s}^{-1}$ (Read \& Pietsch 2001).

More recently, NGC 300 was observed with XMM-Newton on 2000 December 26 during XMM-Newton's revolution 192 and 6 days later during revolution 195 . Some previous results of these observations have been presented by Kendziorra et al. (2001) and Carpano et al. (2004). Data on the luminous supersoft X-ray source XMMU J005510.7-373855 in the center of NGC 300 were presented by Kong \& Di Stefano (2003). In addition to these X-ray data, observations with the $2.2 \mathrm{~m}$ MPG/ESO telescope on La Silla were performed. Here, we use archival images in the broad band $B, V$, and $R$ filters.

In this paper we report a catalog of the NGC 300 X-ray point sources obtained with XMM-Newton data, as well as their optical counterparts. The aim of this work is to present a deeper broad-band catalogue of X-ray selected sources in NGC 300 to facilitate further population studies and searches for counterparts in other wavebands. Detailed studies of selected X-ray sources will be presented elsewhere (Carpano et al., in preparation). The remainder of this work is organized as follows. Section 2 describes the observations and data 
reduction of the X-ray and optical data. In Sect. 3 we describe some global properties of the X-ray point sources detected inside the $D_{25}$ optical disk as well as of NGC 300's central diffuse region. The analysis of the optical counterparts of the X-ray sources is presented in Sect. 4. Tables of the X-ray and optical properties are given in Sect. 5. We discuss our results in Sect. 6.

\section{Observations and data reduction}

\subsection{X-ray observations and data reduction}

XMM-Newton observed NGC 300 during its orbit 192 (2000 December 26; $37 \mathrm{ks}$ on source time) and orbit 195 (2001 January 1; $47 \mathrm{ks}$ on source time). For both observations, all three EPIC cameras were operated in their full frame mode with the medium filter. See Turner et al. (2001) and Strüder et al. (2001) for a description of the EPIC cameras. The aimpoint of the EPIC-pn camera was centered on NGC 300, using the same position as that of the earlier ROSAT data. The goodtime-intervals extracted from the MOS light curve for revolution 192 were also used to filter the events list of the pn-camera, leaving $30 \mathrm{ks}$ of low background data for each of the three cameras. The particle background during revolution 195 was low, resulting in net observing times of $43 \mathrm{ks}$ for the two MOS cameras and $40 \mathrm{ks}$ for the pn-camera.

We reduced the data using the standard XMM-Newton Science Analysis System (SAS), version 6.1.0, using the epchain task for the EPIC-pn and emchain for the MOS cameras. Spectra, images, and lightcurves were extracted using evselect; we only consider events measured in regions away from the CCD borders or bad pixels (FLAG $=0)$, and only single and double events for the pn camera (PATTERN $\leq 4)$ and single to quadruple events for the MOS cameras (PATTERN $\leq$ 12). The Response Matrix and Ancilliary Response files are created with the rmfgen and arfgen tasks using the newest available calibration files.

\subsection{Optical observations and data reduction}

NGC 300 was originally observed between 1999 July and 2000 January with the $2.2 \mathrm{~m} \mathrm{MPG/ESO} \mathrm{telescope} \mathrm{on} \mathrm{La} \mathrm{Silla,}$ Chile, for the ARAUCARIA project (Pietrzyński et al. 2002a), an attempt to fine-tune the cosmic distance ladder by comparing different distance indicators such as Cepheids, blue supergiants, the tip of the red giant branch, and planetary nebulae for various nearby galaxies. The data we used for this work was retrieved from the ESO archive. The reduction was performed in the framework of the Garching-Bonn Deep Survey by Schirmer et al. (2003), who also comment extensively on the data reduction. NGC 300 was observed throughout 34 nights, which resulted in $11 \mathrm{~h}$ (110 images), $10.4 \mathrm{~h}$ (105 images), and $4.2 \mathrm{~h}$ (42 images), in the $B, V$, and $R$ filters, respectively. The observations were centered on $\alpha_{\mathrm{J} 2000.0}=00^{\mathrm{h}} 54^{\mathrm{m}} 50^{\mathrm{s}}, \delta_{\mathrm{J} 2000.0}=$ $-37^{\circ} 40^{\prime} 00^{\prime \prime}$ with a field of view of $34^{\prime} \times 34^{\prime}$. The average seeing in the $B, V$, and $R$ data was $1^{\prime \prime} 1,11^{\prime \prime} 1$ and $1^{\prime \prime} 0$, respectively. The absolute astrometric accuracy of the optical images is $\sim 0.25$ arcsec. The relative astrometry accuracy is about ten times better.

\section{Properties of the X-ray detected sources and the diffuse emission region}

\subsection{Source detection}

Event and attitude file of each instrument were first merged for both orbits 192 and 195, using the SAS merge task. This approach is valid since both observations have the same pointing direction and the difference in position angles between the two observations was very small and consequently the effect of the varying point spread function of XMM-Newton on the resulting image is small. Point source detection was then performed using a maximum likelihood approach as implemented by the SAS-tool edetect_chain. We ran this tool simultaneously on the data from all three cameras, setting a maximum likelihood threshold of 10 in the $0.3-6.0 \mathrm{keV}$ band. After removing sources associated with the cluster of galaxies CL 0053-37, a total of 163 sources were found, of which 86 sources are within the $D_{25}$ optical disk. As it will be shown in Sect. 3.3, our detection limiting flux in the $0.3-6.0 \mathrm{keV}$ energy band is $F_{0.3-6} \sim 7 \times 10^{-16} \mathrm{erg} \mathrm{cm}^{-2} \mathrm{~s}^{-1}$ for sources inside the optical disk.

We adaptively determine source and background regions with the SAS region task, using an elliptical locus to approximate the spatially varying point spread function.

Figure 1 shows the $V$ band optical image of NGC 300 and the contour map of the merged X-ray raw image from both orbits and all three EPIC cameras in the $0.3-6.0 \mathrm{keV}$ energy band. The $D_{25}$ optical disk and the sources detected inside the disk, which are numbered in order of decreasing X-ray count rate as determined by the edetect_chain, are also shown.

A summary of the properties of these detected sources as well as their possible optical counterparts is given in Table 1, described in detail in Sect. 5.

\subsection{Color-color diagram and $X$-ray fluxes}

Any classification of the detected sources as well as the determination of the source flux require an understanding of the spectral shape of the sources. Due to the low count rates of most detected sources, formal spectral modelling is only possible for a few of the brightest sources (these fits will be shown in a subsequent paper). We therefore rely on X-ray color-color and hardness ratio diagrams in the determination of the flux and the spectral shape.

In order to determine these quantities, we first derive the background-subtracted count rate from

$\mathrm{CR}(I)=\frac{C(I)_{\mathrm{src}}}{T_{\mathrm{src}}}-\frac{C(I)_{\mathrm{back}} B_{\mathrm{src}}}{T_{\mathrm{back}} B_{\mathrm{back}}}$

where $C(I)$ is the total number of counts in channel $I, T$ is the exposure time and $B$ is the area from which the source and background data were extracted, as given by the BACKSCAL keyword (see below). The subscripts "src" and "back" denote the source and background, respectively.

The BACKSCAL keyword present in the XMM-SAS produced spectra is defined by the geometric area of the source extraction region minus the bad pixels or CCD gaps laying 


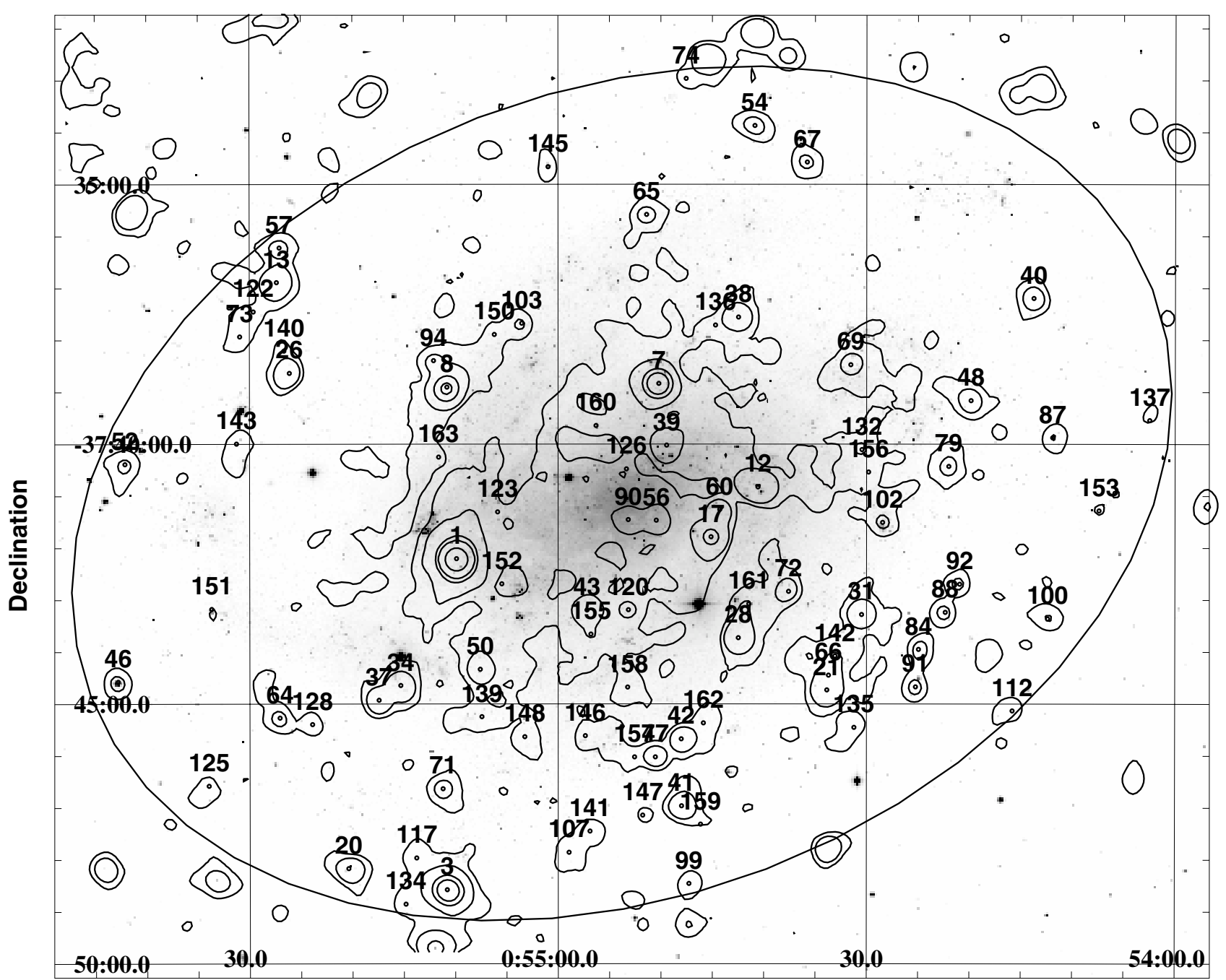

Right ascension

Fig. 1. Optical image of NGC 300 in the visible band overlaid by a contour map of the merged $0.3-6.0 \mathrm{keV}$ raw X-ray image from all three EPIC cameras and from both orbits. The $D_{25}$ optical disk and the sources detected inside the disk are also shown.

within that source region. Due to software bugs, this keyword is not always correctly estimated. Source regions intersecting bad CCD columns often have BACKSCAL overestimated. For that reason, the total number of counts in a given energy band (soft, medium, or hard) in background-subtracted spectra can sometimes be negative. When this happens the data coming from that instrument for that revolution are excluded from the hardness ratio calculation. To obtain the total count rate in each band, we add the valid count rates data from all three EPIC instruments. The X-ray colors are then defined by:

$\mathrm{HR}_{\text {hard }}=\frac{H-M}{H+M+S}, \quad$ and $\quad \mathrm{HR}_{\mathrm{soft}}=\frac{M-S}{H+M+S}$

where $S, M$, and $H$ are the total count rates in the soft $(0.3-1.0 \mathrm{keV})$, medium $(1.0-2.0 \mathrm{keV})$, and hard $(2.0-6.0 \mathrm{keV})$ energy bands. The uncertainty of the hardness ratio and the source countrate is determined by assuming Poisson statistics. Unless otherwise noted, all uncertainties are at the $68 \%$ level.

Figure 2 shows the resulting color-color diagram for the X-ray sources inside the $D_{25}$ optical disk, excluding sources having less than 20 net counts. In Fig. 3 we compare these data with empirical color-color diagrams assuming a simple bremsstrahlung model and a two component source spectrum consisting of a soft bremsstrahlung and a hard power law component (colors derived from simple power law models were not sufficient to describe the data). In these models the equivalent hydrogen column $N_{\mathrm{H}}$, expressed in units of $10^{22} \mathrm{~cm}^{-2}$, is running from 0.03 to 1.0 . In the simple bremsstrahlung model the temperature $k T$ varies from 0.01 to $5.0 \mathrm{keV}$. In the bremsstrahlung plus power-law model the photon index $\Gamma$ varies from 0.5 to 4.5 and the bremsstrahlung temperature is fixed at $0.2 \mathrm{keV}$. Both models are sufficient to describe the data, however, the $N_{\mathrm{H}}$ values inferred are generally larger than the pure Galactic $N_{\mathrm{H}}$ in the direction to NGC 300 (which is $3.6 \times 10^{20} \mathrm{~cm}^{-2}$; Dickey \& Lockman 1990), indicating intrinsic absorption within NGC 300 and also pointing towards a possible contamination of our source sample by background AGN. From the $2-10 \mathrm{keV}$ AGN $\log N$ - $\log S$-diagram of Ueda et al. (2003), $\sim 30$ AGN with $F_{2-10} \geq 10^{-14} \mathrm{erg} \mathrm{cm}^{-2} \mathrm{~s}^{-1}$ are expected within the $D_{25}$-disk, however, the identification of AGN 
Table 1. Summary of the X-ray properties of sources found in NGC 300 (see text for details).

\begin{tabular}{|c|c|c|c|c|c|c|c|c|c|c|}
\hline $\begin{array}{l}\text { ID } \\
(1)\end{array}$ & $\begin{array}{l}\alpha_{\mathrm{J} 2000.0} \\
(2)\end{array}$ & $\begin{array}{l}J 2000.0 \\
3)\end{array}$ & $\begin{array}{l}\text { Pos. err. (") } \\
\text { (4) }\end{array}$ & $\begin{array}{l}\text { Lik. } \\
(5)\end{array}$ & $\begin{array}{l}\text { Counts } \\
(6)\end{array}$ & $\begin{array}{l}\text { Ct. rate } \\
\text { (7) }\end{array}$ & $\begin{array}{l}\text { Hard HR } \\
(8)\end{array}$ & $\begin{array}{l}\text { Soft HR } \\
(9)\end{array}$ & $\begin{array}{l}F_{0.3-6}(\mathrm{cgs}) \\
(10)\end{array}$ & $\begin{array}{l}L_{0.3-6} \text { (cgs) } \\
\text { (11) }\end{array}$ \\
\hline 1 & $00: 55: 10.00$ & $-37: 42: 12.0$ & 0.45 & $5.45 \times 10^{4}$ & $1.53 \pm 0.01 \times 10^{4}$ & $7.39 \pm 0.06 \times 10^{-2}$ & $-0.18 \pm 0.01$ & $-0.29 \pm 0.01$ & $3.49_{-0.12}^{+0.03} \times 10^{-13}$ & $1.70 \times 10^{38}$ \\
\hline 3 & 00:55:10.86 & $-37: 48: 34.4$ & 0.47 & $8.30 \times 10^{3}$ & $3.16 \pm 0.06 \times 10^{3}$ & $1.61 \pm 0.03 \times 10^{-2}$ & $-0.21 \pm 0.01$ & $-0.30 \pm 0.01$ & $7.20_{-0.74}^{+0.33} \times 10^{-14}$ & $3.51 \times 10^{37}$ \\
\hline 7 & 00:54:50.33 & $-37: 38: 49.5$ & 0.47 & $7.43 \times 10^{3}$ & $2.94 \pm 0.06 \times 10^{3}$ & $1.50 \pm 0.03 \times 10^{-2}$ & $-0.14 \pm 0.02$ & $0.28 \pm 0.02$ & $1.06_{-0.05}^{+0.04} \times 10^{-13}$ & $5.20 \times 10^{37}$ \\
\hline 8 & 00:55:10.91 & $-37: 38: 54.3$ & 0.46 & $3.85 \times 10^{3}$ & $1.90 \pm 0.15 \times 10^{2}$ & $4.31 \pm 0.34 \times 10^{-3}$ & $0.01 \pm 0.03$ & $-0.97 \pm 0.03$ & ${ }_{30}^{56} \times 10^{-14}$ & $7.40 \times 10^{36}$ \\
\hline 12 & 00:54:40.66 & $-37: 40: 48.9$ & 0.58 & $1.20 \times 10^{3}$ & $3.85 \pm 0.22 \times 10^{2}$ & $2.83 \pm 0.16 \times 10^{-3}$ & $-0.17 \pm 0.03$ & $-0.58 \pm 0.03$ & ${ }_{9}^{2} \times 10^{-14}$ & $5.03 \times 10^{36}$ \\
\hline 13 & $00: 55: 27.50$ & $-37: 36: 53.5$ & 0.56 & $9.50 \times 10^{2}$ & $5.38 \pm 0.27 \times 10^{2}$ & $2.72 \pm 0.14 \times 10^{-3}$ & $-0.12 \pm 0.04$ & $0.11 \pm 0.04$ & $1.82_{-0.19}^{+0.18} \times 10^{-14}$ & $8.89 \times 10^{36}$ \\
\hline 17 & 00:54:45.22 & $-37: 41: 46.7$ & 0.53 & $1.45 \times 10^{3}$ & $2.73 \pm 0.19 \times 10^{2}$ & $5.26 \pm 0.35 \times 10^{-3}$ & $-0.15 \pm 0.03$ & $-0.67 \pm 0.03$ & $1.91_{-0.32}^{+0.26} \times 10^{-14}$ & $9.32 \times 10^{36}$ \\
\hline 20 & 00:55:20.47 & $-37: 48: 10.2$ & 0.69 & $3.75 \times 10^{2}$ & $2.75 \pm 0.19 \times 10^{2}$ & $1.60 \pm 0.11 \times 10^{-3}$ & $-0.23 \pm 0.04$ & $-0.41 \pm 0.04$ & $6.36_{-1.03}^{+0.98} \times 10^{-15}$ & $3.11 \times 10^{36}$ \\
\hline 21 & 00:54:33.96 & $-37: 44: 43.2$ & 0.58 & $9.17 \times 10^{2}$ & $5.65 \pm 0.27 \times 10^{2}$ & $2.82 \pm 0.14 \times 10^{-3}$ & $-0.13 \pm 0.04$ & $-0.01 \pm 0.04$ & $1.70_{-0.11}^{+0.23} \times 10^{-14}$ & $8.32 \times 10^{36}$ \\
\hline 26 & $00: 55: 26.23$ & $-37: 38: 38.2$ & 0.69 & $4.01 \times 10^{2}$ & $3.01 \pm 0.20 \times 10^{2}$ & $1.57 \pm 0.10 \times 10^{-3}$ & $-0.08 \pm 0.05$ & $0.04 \pm 0.05$ & $\frac{2}{5} \times 10^{-14}$ & $5.29 \times 10^{36}$ \\
\hline 28 & 00:54:42.56 & $-37: 43: 43.2$ & 0.61 & $6.38 \times 10^{2}$ & $3.98 \pm 0.24 \times 10^{2}$ & $1.98 \pm 0.12 \times 10^{-3}$ & $-0.13 \pm 0.05$ & $0.06 \pm 0.05$ & ${ }_{6}^{8} \times 10^{-14}$ & $6.23 \times 10^{36}$ \\
\hline 31 & $00: 54: 30.57$ & $-37: 43: 15.9$ & 0.65 & $5.11 \times 10^{2}$ & $3.26 \pm 0.21 \times 10^{2}$ & $1.67 \pm 0.11 \times 10^{-3}$ & $-0.07 \pm 0.05$ & $0.00 \pm 0.05$ & $\frac{3}{5} \times 10^{-14}$ & $5.58 \times 10^{36}$ \\
\hline 34 & 00:55:15.39 & $-37: 44: 38.9$ & 0.70 & $3.78 \times 10^{2}$ & $6.98 \pm 0.97 \times 10^{1}$ & $9.66 \pm 1.29 \times 10^{-4}$ & $-0.11 \pm 0.08$ & $-0.60 \pm 0.08$ & $3.96_{-0.95}^{+1.97} \times 10^{-15}$ & $1.93 \times 10^{36}$ \\
\hline 37 & 00:55:17.52 & $-37: 44: 55.8$ & 0.66 & $3.82 \times 10^{2}$ & $2.65 \pm 0.19 \times 10^{2}$ & $1.37 \pm 0.10 \times 10^{-3}$ & $-0.27 \pm 0.06$ & $0.09 \pm 0.06$ & $7.41_{-1.21}^{+1.03} \times 10^{-15}$ & $3.62 \times 10^{36}$ \\
\hline 38 & 00:54:42.59 & $-37: 37: 32.7$ & 0.69 & $3.77 \times 10^{2}$ & $2.75 \pm 0.20 \times 10^{2}$ & $1.38 \pm 0.10 \times 10^{-3}$ & $-0.21 \pm 0.05$ & $-0.11 \pm 0.05$ & $\frac{5}{3} \times 10^{-15}$ & $3.40 \times 10^{36}$ \\
\hline 39 & $00: 54: 49.55$ & $-37: 40: 00.7$ & 0.68 & $3.28 \times 10^{2}$ & $2.85 \pm 0.20 \times 10^{2}$ & $1.37 \pm 0.10 \times 10^{-3}$ & $-0.12 \pm 0.06$ & $0.11 \pm 0.06$ & $\frac{1}{5} \times 10^{-15}$ & $4.50 \times 10^{36}$ \\
\hline 40 & 00:54:13.89 & $-37: 37: 10.8$ & 0.76 & $2.17 \times 10^{2}$ & $1.97 \pm 0.17 \times 10^{2}$ & $9.65 \pm 0.83 \times 10^{-4}$ & $-0.18 \pm 0.07$ & $0.15 \pm 0.07$ & ${ }_{6}^{4} \times 10^{-15}$ & $3.03 \times 10^{36}$ \\
\hline 41 & 00:54:48.07 & $-37: 46: 57.4$ & 0.79 & $3.27 \times 10^{2}$ & $2.41 \pm 0.19 \times 10^{2}$ & $1.18 \pm 0.09 \times 10^{-3}$ & $-0.09 \pm 0.06$ & $-0.01 \pm 0.06$ & $7.71_{-1.16}^{+1.35} \times 10^{-15}$ & $3.77 \times 10^{36}$ \\
\hline 42 & 00:54:48.11 & $-37: 45: 40.0$ & 0.69 & $3.71 \times 10^{2}$ & $2.62 \pm 0.19 \times 10^{2}$ & $1.26 \pm 0.09 \times 10^{-3}$ & $-0.09 \pm 0.06$ & $0.12 \pm 0.06$ & $8.82_{-1.27}^{+1.46} \times 10^{-15}$ & $4.30 \times 10^{36}$ \\
\hline 43 & 00:54:57.28 & $-37: 43: 11.4$ & 0.69 & $3.38 \times 10^{2}$ & $2.81 \pm 0.20 \times 10^{2}$ & $1.41 \pm 0.10 \times 10^{-3}$ & $-0.09 \pm 0.06$ & $0.06 \pm 0.06$ & $\times 10^{-15}$ & $4.76 \times 10^{36}$ \\
\hline 46 & 00:55:42.91 & $-37: 44: 34.8$ & 0.96 & $1.33 \times 10^{2}$ & $6.77 \pm 1.05 \times 10^{1}$ & $5.89 \pm 0.88 \times 10^{-4}$ & $-0.01 \pm 0.09$ & $-0.61 \pm 0.09$ & $\times 10^{-15}$ & $1.61 \times 10^{36}$ \\
\hline 47 & $00: 54: 50.63$ & $-37: 46: 00.7$ & 0.69 & $2.51 \times 10^{2}$ & $1.99 \pm 0.17 \times 10^{2}$ & $9.48 \pm 0.80 \times 10^{-4}$ & $-0.02 \pm 0.07$ & $0.09 \pm 0.07$ & $\times 10^{-15}$ & $3.51 \times 10^{36}$ \\
\hline 48 & 00:54:19.97 & $-37: 39: 08.9$ & 0.87 & $1.63 \times 10^{2}$ & $1.61 \pm 0.16 \times 10^{2}$ & $7.16 \pm 0.73 \times 10^{-4}$ & $-0.12 \pm 0.09$ & $0.32 \pm 0.09$ & $73 \times 10^{-15}$ & $2.62 \times 10^{36}$ \\
\hline 50 & 00:55:07.67 & $-37: 44: 20.1$ & 0.76 & $2.13 \times 10^{2}$ & $2.01 \pm 0.17 \times 10^{2}$ & $1.01 \pm 0.09 \times 10^{-3}$ & $0.01 \pm 0.08$ & $0.20 \pm 0.08$ & $18 \times 10^{-15}$ & $4.07 \times 10^{36}$ \\
\hline 52 & $00: 55: 42.24$ & $-37: 40: 23.7$ & 1.13 & $9.98 \times 10^{1}$ & $.52 \pm 1.03 \times 10^{1}$ & $1.14 \pm 0.17 \times 10^{-3}$ & $-0.01 \pm 0.07$ & $-0.86 \pm 0.07$ & $5.08_{-1.38}^{+2.02} \times 10^{-15}$ & $2.48 \times 10^{36}$ \\
\hline 54 & 00:54:41.04 & $-37: 33: 51.7$ & 0.99 & $1.39 \times 10^{2}$ & $1.46 \pm 0.14 \times 10^{2}$ & $7.92 \pm 0.81 \times 10^{-4}$ & $0.35 \pm 0.10$ & $0.12 \pm 0.10$ & $8 \times 10^{-15}$ & $4.38 \times 10^{36}$ \\
\hline 56 & $00: 54: 50.56$ & $-37: 41: 27.8$ & 0.78 & $2.16 \times 10^{2}$ & $1.37 \pm 0.14 \times 10^{2}$ & $8.59 \pm 0.91 \times 10^{-4}$ & $-0.16 \pm 0.07$ & $-0.44 \pm 0.07$ & $47 \times 10^{-15}$ & $1.77 \times 10^{36}$ \\
\hline 57 & $00: 55: 27.24$ & $-37: 36: 13.5$ & 1.06 & $9.43 \times 10^{1}$ & $1.10 \pm 0.13 \times 10^{2}$ & $6.76 \pm 0.79 \times 10^{-4}$ & $-0.17 \pm 0.09$ & $0.06 \pm 0.09$ & $4.19_{-0.95}^{+1.03} \times 10^{-15}$ & $2.05 \times 10^{36}$ \\
\hline 60 & 00:54:44.38 & $-37: 41: 14.7$ & 0.78 & $1.65 \times 10^{2}$ & $8.66 \pm 1.15 \times 10^{1}$ & $1.20 \pm 0.16 \times 10^{-3}$ & $-0.16 \pm 0.07$ & $-0.51 \pm 0.07$ & $5.01_{-1.30}^{+1.58} \times 10^{-15}$ & $2.45 \times 10^{36}$ \\
\hline 64 & $00: 55: 27.16$ & $-37: 45: 16.5$ & 0.95 & $1.04 \times 10^{2}$ & $.02 \pm 0.13 \times 10^{2}$ & $6.52 \pm 0.78 \times 10^{-4}$ & $-0.33 \pm 0.10$ & $0.08 \pm 0.10$ & $76 \times 10^{-15}$ & $1.56 \times 10^{36}$ \\
\hline 65 & 00:54:51.52 & $-37: 35: 34.5$ & 0.98 & $1.14 \times 10^{2}$ & $1.01 \pm 0.12 \times 10^{2}$ & $7.63 \pm 0.94 \times 10^{-4}$ & $-0.07 \pm 0.10$ & $0.02 \pm 0.10$ & $5.22_{-1.18}^{+1.46} \times 10^{-15}$ & $2.55 \times 10^{36}$ \\
\hline 66 & 00:54:33.79 & $-37: 44: 26.1$ & 0.94 & $8.00 \times 10^{1}$ & $2.01 \pm 0.17 \times 10^{2}$ & $9.44 \pm 0.84 \times 10^{-4}$ & $-0.07 \pm 0.08$ & $0.35 \pm 0.08$ & $7.49_{-1.32}^{+1.45} \times 10^{-15}$ & $3.66 \times 10^{36}$ \\
\hline 67 & $00: 54: 35.93$ & $-37: 34: 33.8$ & 1.02 & $8.91 \times 10^{1}$ & $1.02 \pm 0.13 \times 10^{2}$ & $5.10 \pm 0.64 \times 10^{-4}$ & $-0.03 \pm 0.10$ & $0.05 \pm 0.10$ & $3.68_{-0.83}^{+0.99} \times 10^{-15}$ & $1.80 \times 10^{36}$ \\
\hline 69 & 00:54:31.67 & $-37: 38: 27.7$ & 0.98 & $9.87 \times 10^{1}$ & $9.22 \pm 1.31 \times 10^{1}$ & $4.55 \pm 0.64 \times 10^{-4}$ & $-0.17 \pm 0.11$ & $0.12 \pm 0.11$ & $2.88_{-0.77}^{+0.90} \times 10^{-15}$ & $1.40 \times 10^{36}$ \\
\hline 71 & $00: 55: 11.28$ & $-37: 46: 37.7$ & 0.97 & $1.01 \times 10^{2}$ & $9.27 \pm 1.24 \times 10^{1}$ & $5.75 \pm 0.74 \times 10^{-4}$ & $-0.18 \pm 0.12$ & $0.43 \pm 0.12$ & $4.18_{-1.08}^{+1.08} \times 10^{-15}$ & $2.04 \times 10^{36}$ \\
\hline 72 & 00:54:37.68 & $-37: 42: 49.6$ & 0.92 & $1.29 \times 10^{2}$ & $1.34 \pm 0.14 \times 10^{2}$ & $6.91 \pm 0.73 \times 10^{-4}$ & $-0.09 \pm 0.08$ & $-0.01 \pm 0.08$ & $4.53_{-0.86}^{+1.17} \times 10^{-15}$ & $2.21 \times 10^{36}$ \\
\hline 73 & 00:55:31.08 & $-37: 37: 56.1$ & 1.20 & $5.32 \times 10^{1}$ & $6.68 \pm 1.01 \times 10^{1}$ & $4.32 \pm 0.63 \times 10^{-4}$ & $-0.05 \pm 0.12$ & $0.10 \pm 0.12$ & $3.14_{-0.87}^{+1.10} \times 10^{-15}$ & $1.53 \times 10^{36}$ \\
\hline 74 & 00:54:47.71 & $-37: 32: 57.5$ & 1.45 & & $4.24 \pm 0.87 \times 10^{1}$ & $3.33 \pm 0.63 \times 10^{-4}$ & $0.05 \pm 0.15$ & $-0.06 \pm 0.15$ & $2.56_{-0.86}^{+1.04} \times 10^{-15}$ & $1.25 \times 10^{36}$ \\
\hline 79 & $00: 54: 22.13$ & $-37: 40: 25.0$ & 1.05 & $9.44 \times 10^{1}$ & $4.04 \pm 3.11 \times 10^{0}$ & $1.79 \pm 1.03 \times 10^{-4}$ & & & & \\
\hline 84 & $00: 54: 25.03$ & $-37: 43: 56.3$ & 1.04 & $8.13 \times 10^{1}$ & $5.38 \pm 0.93 \times 10^{1}$ & $3.69 \pm 0.72 \times 10^{-4}$ & $0.27 \pm 0.19$ & $0.31 \pm 0.19$ & $3.99_{-1.25}^{+1.37} \times 10^{-15}$ & $1.95 \times 10^{36}$ \\
\hline 87 & $00: 54: 12.03$ & $-37: 39: 51.7$ & 1.08 & $6.42 \times 10^{1}$ & $8.52 \pm 1.14 \times 10^{1}$ & $4.73 \pm 0.65 \times 10^{-4}$ & $-0.19 \pm 0.12$ & $0.20 \pm 0.12$ & $3.09_{-0.88}^{+1.01} \times 10^{-15}$ & $1.51 \times 10^{36}$ \\
\hline 88 & 00:54:22.44 & $-37: 43: 13.3$ & 1.02 & $9.19 \times 10^{1}$ & $3.74 \pm 0.81 \times 10^{1}$ & $6.33 \pm 1.18 \times 10^{-4}$ & $-0.08 \pm 0.12$ & $-0.41 \pm 0.12$ & $3.24_{-1.19}^{+1.76} \times 10^{-15}$ & $1.58 \times 10^{36}$ \\
\hline 90 & 00:54:53.28 & $-37: 41: 27.0$ & 0.95 & $8.94 \times 10^{1}$ & $9.37 \pm 1.27 \times 10^{1}$ & $5.80 \pm 0.78 \times 10^{-4}$ & $-0.16 \pm 0.10$ & $-0.06 \pm 0.10$ & $3.37_{-0.86}^{+1.06} \times 10^{-15}$ & $1.65 \times 10^{36}$ \\
\hline 91 & 00:54:25.32 & $-37: 44: 39.5$ & 1.11 & $8.24 \times 10^{1}$ & $8.25 \pm 1.17 \times 10^{1}$ & $6.29 \pm 0.87 \times 10^{-4}$ & $-0.14 \pm 0.11$ & $-0.07 \pm 0.11$ & $3.66_{-0.95}^{+1.16} \times 10^{-15}$ & $1.79 \times 10^{36}$ \\
\hline 92 & 00:54:21.04 & $-37: 42: 40.8$ & 1.12 & $6.19 \times 10^{1}$ & $4.58 \pm 0.83 \times 10^{1}$ & $3.89 \pm 0.76 \times 10^{-4}$ & $0.23 \pm 0.18$ & $0.18 \pm 0.18$ & $4.04_{-1.36}^{+1.63} \times 10^{-15}$ & $1.97 \times 10^{36}$ \\
\hline 94 & $00: 55: 12.23$ & $-37: 38: 23.8$ & 1.10 & $5.98 \times 10^{1}$ & $7.99 \pm 1.16 \times 10^{1}$ & $5.85 \pm 0.88 \times 10^{-4}$ & $-0.09 \pm 0.10$ & $-0.33 \pm 0.10$ & $3.07_{-0.90}^{+1.12} \times 10^{-15}$ & $1.50 \times 10^{36}$ \\
\hline 99 & 00:54:47.35 & $-37: 48: 26.9$ & 1.13 & $5.72 \times 10^{1}$ & $5.39 \pm 0.97 \times 10^{1}$ & $4.23 \pm 0.73 \times 10^{-4}$ & $-0.05 \pm 0.15$ & $0.17 \pm 0.15$ & $3.21_{-1.02}^{+1.23} \times 10^{-15}$ & $1.57 \times 10^{36}$ \\
\hline 100 & $00: 54: 12.47$ & $-37: 43: 20.7$ & 1.23 & $4.86 \times 10^{1}$ & $6.01 \pm 1.03 \times 10^{1}$ & $3.26 \pm 0.53 \times 10^{-4}$ & $-0.31 \pm 0.14$ & $0.19 \pm 0.14$ & $1.77_{-0.59}^{+0.70} \times 10^{-15}$ & $8.64 \times 10^{35}$ \\
\hline 102 & $00: 54: 28.55$ & $-37: 41: 29.7$ & 1.04 & $6.83 \times 10^{1}$ & $1.09 \pm 0.39 \times 10^{1}$ & $3.53 \pm 1.27 \times 10^{-4}$ & & & & \\
\hline 103 & 00:55:03.66 & $-37: 37: 40.1$ & 1.00 & $6.14 \times 10^{1}$ & $8.42 \pm 1.11 \times 10^{1}$ & $4.08 \pm 0.57 \times 10^{-4}$ & $0.00 \pm 0.09$ & $-0.42 \pm 0.09$ & $2.36_{-0.62}^{+0.88} \times 10^{-15}$ & $1.15 \times 10^{36}$ \\
\hline 107 & 00:54:59.02 & $-37: 47: 51.0$ & 1.42 & $4.69 \times 10^{1}$ & $5.35 \pm 0.96 \times 10^{1}$ & $4.01 \pm 0.70 \times 10^{-4}$ & $0.34 \pm 0.17$ & $0.24 \pm 0.17$ & $4.58_{-1.31}^{+1.46} \times 10^{-15}$ & $2.23 \times 10^{36}$ \\
\hline
\end{tabular}


Table 1. continued.

\begin{tabular}{|c|c|c|c|c|c|c|c|c|c|c|}
\hline $\begin{array}{l}\text { ID } \\
(1)\end{array}$ & $\begin{array}{l}\alpha_{\mathrm{J} 2000.0} \\
(2)\end{array}$ & $\begin{array}{l}\delta_{\mathrm{J} 2000.0} \\
(3)\end{array}$ & $\begin{array}{l}\text { Pos. err. (") } \\
\text { (4) }\end{array}$ & $\begin{array}{l}\text { Lik. } \\
(5)\end{array}$ & $\begin{array}{l}\text { Counts } \\
(6)\end{array}$ & $\begin{array}{l}\text { Ct. rate } \\
\text { (7) }\end{array}$ & $\begin{array}{l}\text { Hard HR } \\
(8)\end{array}$ & $\begin{array}{l}\text { Soft HR } \\
\text { (9) }\end{array}$ & $\begin{array}{l}F_{0.3-6}(\mathrm{cgs}) \\
(10)\end{array}$ & $\begin{array}{l}L_{0.3-6}(\mathrm{cgs}) \\
(11)\end{array}$ \\
\hline 112 & $00: 54: 15.91$ & $-37: 45: 07.0$ & 1.42 & $4.45 \times 10^{1}$ & $4.59 \pm 0.86 \times 10^{1}$ & $4.61 \pm 0.86 \times 10^{-4}$ & $-0.03 \pm 0.09$ & $-0.74 \pm 0.09$ & $2.26_{-0.99}^{+0.71} \times 10^{-15}$ & $1.10 \times 10^{36}$ \\
\hline 117 & 00:55:13.85 & $-37: 47: 57.8$ & 1.71 & $2.49 \times 10^{1}$ & $3.39 \pm 0.77 \times 10^{1}$ & $3.04 \pm 0.71 \times 10^{-4}$ & $-0.08 \pm 0.19$ & $0.19 \pm 0.19$ & $2.23_{-0.86}^{+1.26} \times 10^{-15}$ & $1.09 \times 10^{36}$ \\
\hline 120 & 00:54:53.26 & $-37: 43: 10.8$ & 1.07 & $5.21 \times 10^{1}$ & $4.63 \pm 0.85 \times 10^{1}$ & $3.85 \pm 0.74 \times 10^{-4}$ & $0.40 \pm 0.17$ & $-0.31 \pm 0.17$ & & \\
\hline 122 & $00: 55: 29.75$ & $-37: 37: 27.4$ & 1.65 & $1.80 \times 10^{1}$ & $1.14 \pm 0.49 \times 10^{1}$ & $1.58 \pm 0.64 \times 10^{-4}$ & & & & \\
\hline 123 & 00:55:06.00 & $-37: 41: 18.1$ & 1.62 & $2.37 \times 10^{1}$ & $5.79 \pm 3.03 \times 10^{0}$ & $1.74 \pm 0.87 \times 10^{-4}$ & & & & \\
\hline 125 & 00:55:34.06 & $-37: 46: 35.1$ & 1.66 & $1.81 \times 10^{1}$ & $2.61 \pm 0.65 \times 10^{1}$ & $2.26 \pm 0.62 \times 10^{-4}$ & $-0.21 \pm 0.24$ & $0.32 \pm 0.24$ & $1.51_{-0.69}^{+1.05} \times 10^{-15}$ & $7.40 \times 10^{35}$ \\
\hline 126 & 00:54:53.47 & $-37: 40: 28.3$ & 1.42 & $3.10 \times 10^{1}$ & $2.46 \pm 0.65 \times 10^{1}$ & $2.27 \pm 0.59 \times 10^{-4}$ & $-0.10 \pm 0.16$ & $-0.53 \pm 0.16$ & $1.04_{-0.48}^{+0.81} \times 10^{-15}$ & $5.09 \times 10^{35}$ \\
\hline 128 & 00:55:23.99 & $-37: 45: 24.1$ & 1.51 & $2.41 \times 10^{1}$ & $3.23 \pm 0.74 \times 10^{1}$ & $2.25 \pm 0.54 \times 10^{-4}$ & $0.18 \pm 0.21$ & $0.13 \pm 0.21$ & $2.19_{-0.89}^{+1.06} \times 10^{-15}$ & $1.07 \times 10^{36}$ \\
\hline 132 & 00:54:30.56 & $-37: 40: 05.4$ & 1.39 & $3.09 \times 10^{1}$ & $2.89 \pm 0.72 \times 10^{1}$ & $3.02 \pm 0.96 \times 10^{-4}$ & $0.48 \pm 0.31$ & $0.15 \pm 0.31$ & $3.87_{-1.88}^{+1.49} \times 10^{-15}$ & $1.89 \times 10^{36}$ \\
\hline 134 & $00: 55: 14.88$ & $-37: 48: 51.2$ & 1.87 & $1.25 \times 10^{1}$ & $1.54 \pm 0.56 \times 10^{1}$ & $1.29 \pm 0.49 \times 10^{-4}$ & & & & \\
\hline 135 & 00:54:31.31 & $-37: 45: 26.2$ & 1.44 & $2.91 \times 10^{1}$ & $4.52 \pm 2.89 \times 10^{0}$ & $1.85 \pm 0.96 \times 10^{-4}$ & & & & \\
\hline 136 & $00: 54: 44.82$ & $-37: 37: 42.1$ & 1.38 & $2.07 \times 10^{1}$ & $2.12 \pm 0.62 \times 10^{1}$ & $1.81 \pm 0.55 \times 10^{-4}$ & $-0.16 \pm 0.24$ & $0.20 \pm 0.24$ & $1.22_{-0.61}^{+0.88} \times 10^{-15}$ & $5.97 \times 10^{35}$ \\
\hline 137 & 00:54:02.59 & $-37: 39: 31.4$ & 1.96 & $1.05 \times 10^{1}$ & $1.09 \pm 0.42 \times 10^{1}$ & $1.68 \pm 0.66 \times 10^{-4}$ & & & & \\
\hline 139 & 00:55:07.51 & $-37: 45: 14.6$ & 1.61 & $2.24 \times 10^{1}$ & $1.97 \pm 0.61 \times 10^{1}$ & $2.15 \pm 0.58 \times 10^{-4}$ & & & & \\
\hline 140 & $00: 55: 26.77$ & $-37: 38: 12.7$ & 1.84 & $1.40 \times 10^{1}$ & $1.75 \pm 0.52 \times 10^{1}$ & $1.64 \pm 0.44 \times 10^{-4}$ & & & & \\
\hline 141 & 00:54:56.97 & $-37: 47: 26.7$ & 1.49 & $2.47 \times 10^{1}$ & $1.47 \pm 0.50 \times 10^{1}$ & $3.11 \pm 0.94 \times 10^{-4}$ & & & & \\
\hline 142 & $00: 54: 33.17$ & $-37: 44: 03.9$ & 1.78 & $1.85 \times 10^{1}$ & $1.15 \pm 0.45 \times 10^{1}$ & $1.33 \pm 0.56 \times 10^{-4}$ & & & & \\
\hline 143 & 00:55:31.39 & $-37: 40: 00.1$ & 1.87 & $1.44 \times 10^{1}$ & $1.13 \pm 0.39 \times 10^{1}$ & $1.13 \pm 0.47 \times 10^{-4}$ & & & & \\
\hline 145 & 00:55:01.11 & $-37: 34: 39.4$ & 2.96 & $1.19 \times 10^{1}$ & $6.33 \pm 3.98 \times 10^{0}$ & $1.18 \pm 0.66 \times 10^{-4}$ & & & & \\
\hline 146 & $00: 54: 57.44$ & $-37: 45: 36.3$ & 1.49 & $2.57 \times 10^{1}$ & $3.43 \pm 0.77 \times 10^{1}$ & $2.33 \pm 0.48 \times 10^{-4}$ & $0.00 \pm 0.16$ & $-0.01 \pm 0.16$ & $1.74_{-0.64}^{+0.74} \times 10^{-15}$ & $8.48 \times 10^{35}$ \\
\hline 147 & 00:54:51.84 & $-37: 47: 08.2$ & 1.76 & $1.75 \times 10^{1}$ & $2.63 \pm 0.62 \times 10^{1}$ & $1.93 \pm 0.47 \times 10^{-4}$ & $-0.23 \pm 0.19$ & $-0.09 \pm 0.19$ & $1.01_{-0.47}^{+0.61} \times 10^{-15}$ & $4.93 \times 10^{35}$ \\
\hline 148 & 00:55:03.32 & $-37: 45: 37.6$ & 1.79 & $1.74 \times 10^{1}$ & $2.43 \pm 0.68 \times 10^{1}$ & $1.40 \pm 0.41 \times 10^{-4}$ & $-0.09 \pm 0.20$ & $-0.34 \pm 0.20$ & $7.09_{-3.30}^{+5.97} \times 10^{-16}$ & $3.46 \times 10^{35}$ \\
\hline 150 & 00:55:06.32 & $-37: 37: 53.2$ & 1.78 & $1.51 \times 10^{1}$ & $1.59 \pm 0.57 \times 10^{1}$ & $1.44 \pm 0.56 \times 10^{-4}$ & & & & \\
\hline 151 & 00:55:33.79 & $-37: 43: 11.0$ & 2.74 & $1.01 \times 10^{1}$ & $0.00 \pm 0.00 \times 10^{0}$ & & & & & \\
\hline 152 & 00:55:05.57 & $-37: 42: 41.4$ & 1.79 & $1.07 \times 10^{1}$ & $1.43 \pm 0.56 \times 10^{1}$ & $1.68 \pm 0.63 \times 10^{-4}$ & & & & \\
\hline 153 & 00:54:07.51 & $-37: 41: 15.6$ & 2.10 & $1.02 \times 10^{1}$ & $0.00 \pm 0.00 \times 10^{0}$ & & & & & \\
\hline 155 & 00:54:56.89 & $-37: 43: 39.3$ & 1.60 & $1.63 \times 10^{1}$ & $2.16 \pm 0.62 \times 10^{1}$ & $1.38 \pm 0.38 \times 10^{-4}$ & $-0.16 \pm 0.22$ & $-0.07 \pm 0.22$ & $7.73_{-3.69}^{+5.59} \times 10^{-16}$ & $3.77 \times 10^{35}$ \\
\hline 156 & 00:54:29.92 & $-37: 40: 31.5$ & 1.55 & $1.46 \times 10^{1}$ & $1.21 \pm 0.44 \times 10^{1}$ & $1.49 \pm 0.50 \times 10^{-4}$ & & & & \\
\hline 157 & $00: 54: 52.65$ & $-37: 46: 00.7$ & 2.23 & $1.13 \times 10^{1}$ & $0.00 \pm 0.00 \times 10^{0}$ & & & & & \\
\hline 158 & 00:54:53.33 & $-37: 44: 40.1$ & 1.74 & $1.30 \times 10^{1}$ & $1.25 \pm 0.49 \times 10^{1}$ & $8.99 \pm 3.67 \times 10^{-5}$ & & & & \\
\hline 159 & $00: 54: 46.21$ & $-37: 47: 18.6$ & 1.68 & $1.45 \times 10^{1}$ & $8.42 \pm 3.97 \times 10^{0}$ & $9.87 \pm 4.78 \times 10^{-5}$ & & & & \\
\hline 160 & $00: 54: 56.43$ & $-37: 39: 38.1$ & 2.03 & $1.19 \times 10^{1}$ & $4.42 \pm 3.09 \times 10^{0}$ & $1.33 \pm 0.83 \times 10^{-4}$ & & & & \\
\hline 161 & $00: 54: 41.54$ & $-37: 43: 03.9$ & 2.18 & $1.35 \times 10^{1}$ & $3.55 \pm 3.28 \times 10^{0}$ & $8.06 \pm 11.10 \times 10^{-5}$ & & & & \\
\hline 162 & 00:54:45.95 & $-37: 45: 21.6$ & 1.54 & $1.27 \times 10^{1}$ & $1.98 \pm 0.54 \times 10^{1}$ & $1.45 \pm 0.52 \times 10^{-4}$ & & & & \\
\hline 163 & $00: 55: 11.74$ & $-37: 40: 14.8$ & 1.69 & $1.08 \times 10^{1}$ & $2.71 \pm 2.30 \times 10^{0}$ & $7.65 \pm 5.27 \times 10^{-5}$ & & & & \\
\hline
\end{tabular}

in our sample requires X-ray spectral analysis which is only possible for the brightest sources and dangerous in itself due to the similarity of AGN and XRB spectra.

Our color-color diagram analysis shows that for all sources except one it is possible to find a best matching bremsstrahlung or bremsstrahlung plus power law model. The spectrum of the one non-matching source (\#120), which is in a very complex region, is peculiar and has been excluded from the subsequent analysis. The low number of counts precludes any further statement about the nature of this source.

From this best matching spectral model it is then possible to determine the flux of a source by appropriately scaling the flux determined from the spectral model to the source count rate. The uncertainty of the flux is derived from the minimum and maximum value of fluxes as determined from the error box of the color-color space defined by the source colors. Note that such an approach gives generally more believable flux estimates than the more commonly used approach of assuming one fixed spectral shape for all detected sources, while not limiting one to determining spectral fluxes only for sources with sufficient counts to enable formal spectral model fitting (see also Humphrey \& Buote 2004).

Figure 4 shows the source fluxes versus both hardness ratios defined by Eq. (2). For comparison, a source with a luminosity of $1.82 \times 10^{38} \mathrm{erg} \mathrm{s}^{-1}$, close to the Eddington limit for a $1.4 M_{\odot}$ object, has an integral flux of $3.73 \times 10^{-13} \mathrm{erg} \mathrm{cm}^{-2} \mathrm{~s}^{-1}$ at the distance of NGC 300.

Our brightest source, source 1 , is found with a luminosity of $1.70 \times 10^{38} \mathrm{erg} \mathrm{s}^{-1}$ which is very close to the Eddington limit for a $1.4 M_{\odot}$ object. The source, coincident with the previously known ROSAT source P42 (Read \& Pietsch 2001), has been found to have a slightly lower luminosity than in the ROSAT 


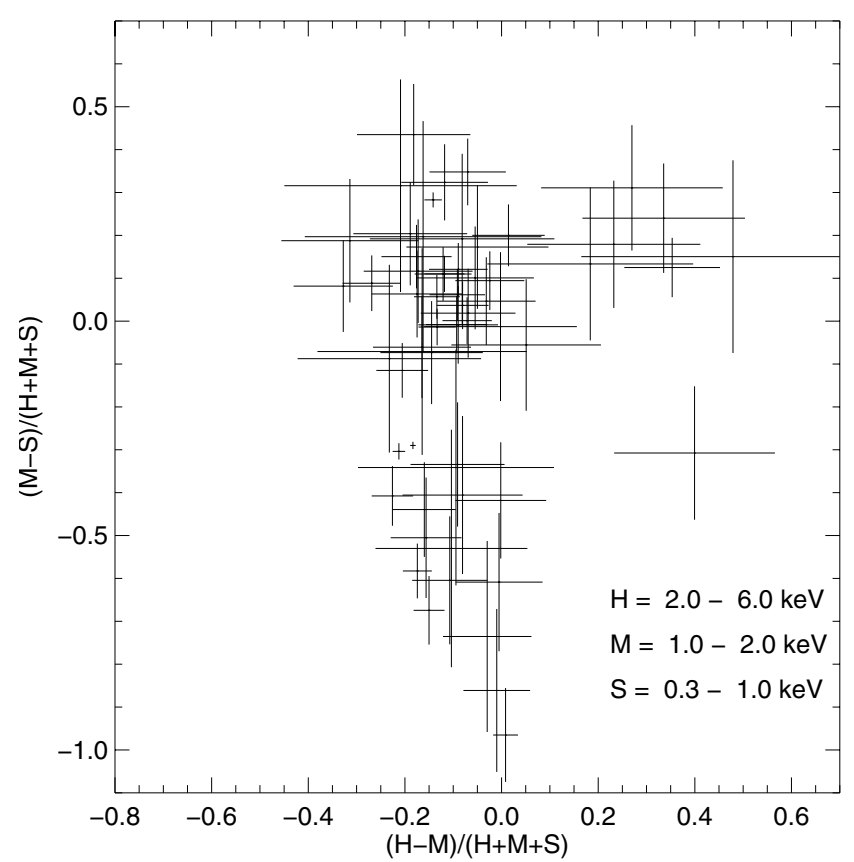

Fig. 2. Color-color diagram of sources detected inside the $D_{25}$ optical disk.

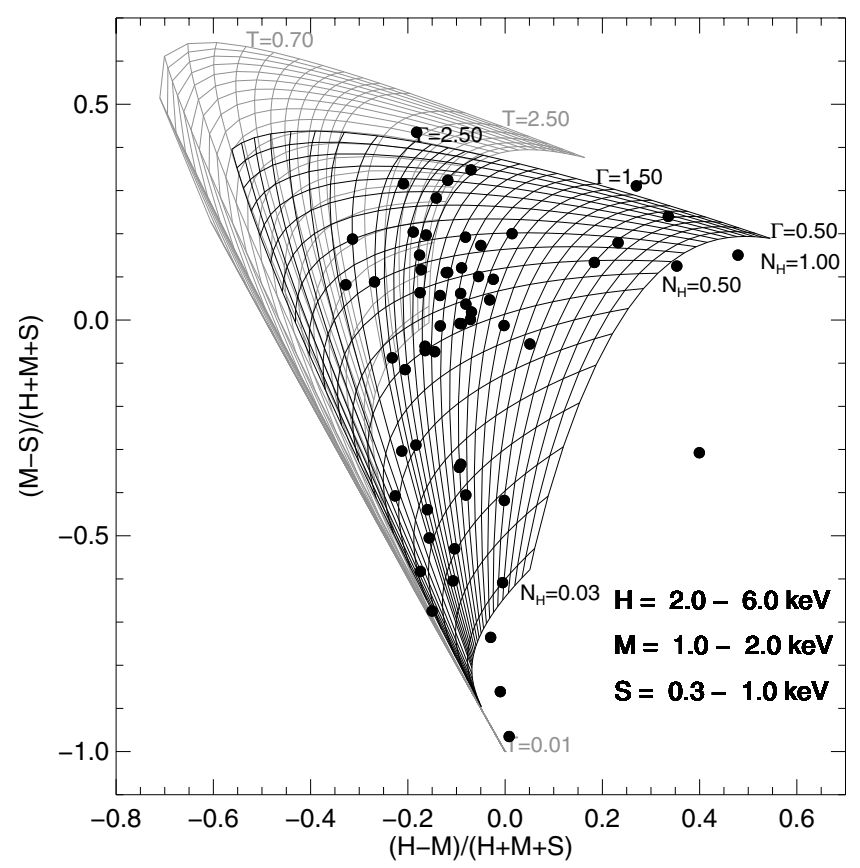

Fig. 3. Color-color diagram of the sources detected inside the $D_{25}$ optical disk and color-color contours for bremsstrahlung and $0.2 \mathrm{keV}$ bremsstrahlung plus power law component. The equivalent hydrogen column, $N_{\mathrm{H}}$, is given in units of $10^{22} \mathrm{~cm}^{-2}$, the temperature of the bremsstrahlung spectrum, $k T$, is given in $\mathrm{keV}$.

observations, but spectral fitting of the brightest X-ray sources, which will be given in a forthcoming paper, is needed to certify if these sources have an intrinsic variability. From their high intrinsic luminosities, these sources are akin to $\sim 10 M_{\odot}$ black holes in their soft state such as LMC X-1 or LMC X-3 (Nowak et al. 2001; Wilms et al. 2001). There are no clear super-Eddington X-ray sources detected in NGC 300. Finally,

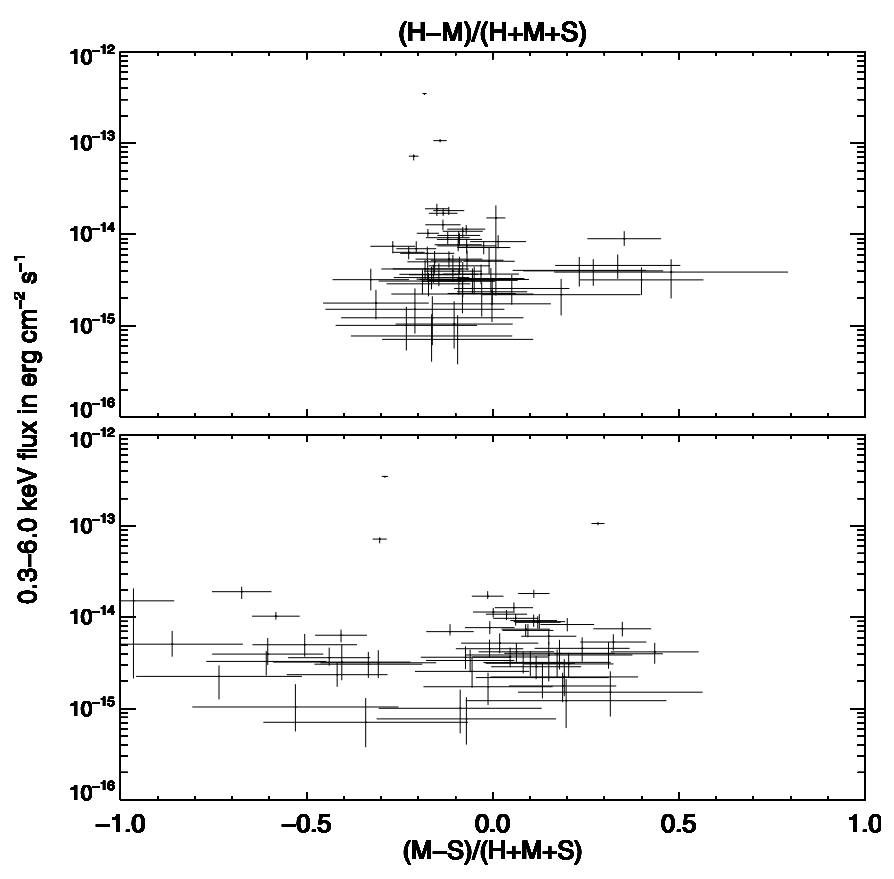

Fig. 4. Fluxes of the sources detected inside the $D_{25}$ optical disk as a function of the harder (top) and softer (bottom) hardness ratio defined by Eq. (2).

we also note that both hardness ratios do not depend significantly on flux.

\subsection{The luminosity function of NGC 300}

Figure 5 shows the $\log N-\log S$ diagram for all detected sources that are inside the optical disk of NGC 300 and having more than 20 net counts, expressed as a function of their X-ray luminosity and flux. Note that we do not make an attempt to correct for possible background AGN, which could appear as sources which are strongly absorbed by the gas within NGC 300.

The break of the power law at a luminosity of $1.5 \times$ $10^{36} \mathrm{erg} \mathrm{s}^{-1}$ (corresponding to $F_{0.3-6} \sim 3 \times 10^{-15} \mathrm{erg} \mathrm{cm}^{-2} \mathrm{~s}^{-1}$ ) defines our completeness limit. Describing the luminosity function above this limit by a pure powerlaw, $N \propto L^{-\alpha}$, we use a Maximum-Likelihood method in the form suggested by Crawford et al. (1970). We find a slope of $\alpha=1.17 \pm 0.17$ (since our source sample is lacking objects with $L_{\mathrm{X}} \gg$ $10^{38} \mathrm{erg} \mathrm{s}^{-1}$, such a simple power law Ansatz for the luminosity function is sufficient; see, e.g., Humphrey \& Buote 2004). This slope of the NGC 300 luminosity function is similar to the slope of the disk population in several other nearby spirals such as M $31(\alpha=0.9 \pm 0.1$; Williams et al. 2004) or NGC 1332 (Humphrey \& Buote 2004), and also in agreement with the mean slope for nearby spiral galaxies $(\alpha=0.79 \pm 0.24$; Colbert et al. 2004).

Due to the apparent bimodality of the sources in Fig. 2, we define two subclasses of sources: hard sources, defined by $\mathrm{HR}_{\text {soft }}>-0.2$, and soft sources, with $\mathrm{HR}_{\text {soft }} \leq-0.2$. Fitting a pure power law to both curves, we find a slope of $1.12 \pm 0.27$ and $1.23 \pm 0.22$ for the soft and the hard sources respectively. Excluding sources above a limiting luminosity of 


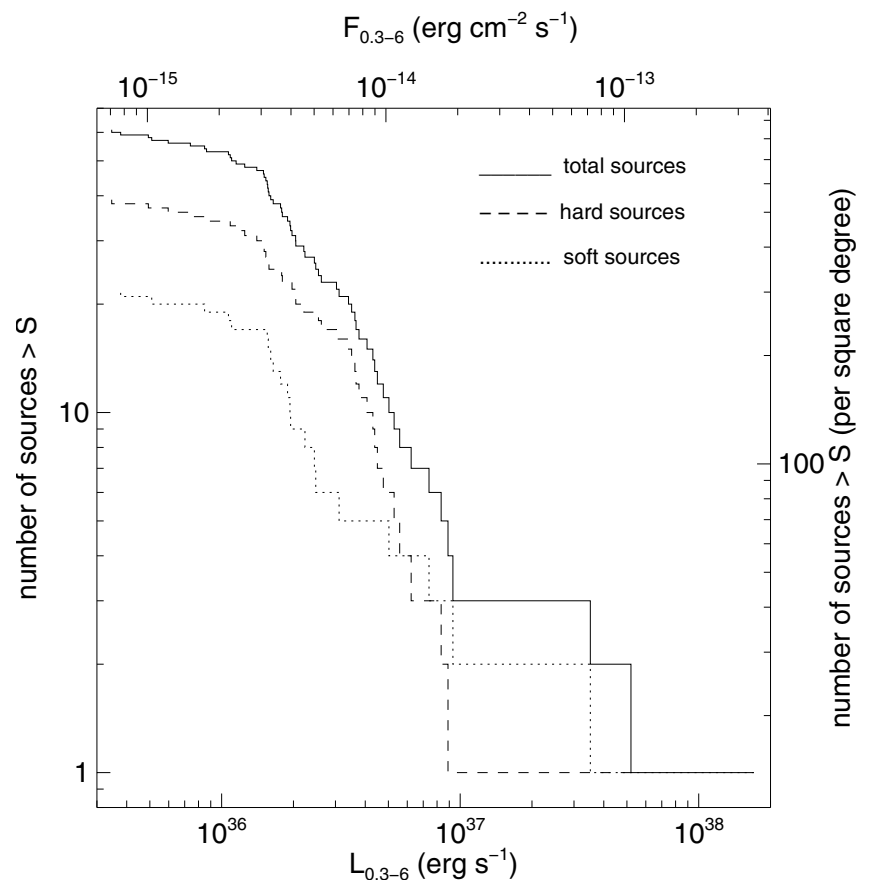

Fig. 5. $\log N-\log S$ diagram of all sources with more than 20 net counts inside the optical $D_{25}$ disk (solid line), of soft sources with $\mathrm{HR}_{\text {soft }} \leq-0.2$ (dotted line), and for sources harder than that (dashed line).

$1 \times 10^{37} \mathrm{erg} \mathrm{s}^{-1}$ (excluding two sources in the soft and one in the hard sample), the Maximum-Likelihood method gives a slope of $\alpha_{\text {soft }}=2.03 \pm 0.52$ and $\alpha_{\text {hard }}=1.39 \pm 0.26$ for the soft and the hard sources respectively. The soft power law slope found here is a bit higher than that of the Milky Way HMXB (Grimm et al. 2002, finding a slope of $\alpha=0.6_{-0.12}^{+0.14}$ ). The shape of the hard sources is instead more complex. Grimm et al. (2002) described the Milky Way LMXB luminosity function by a modified power law which takes into account the gradual steepening of the $\log N-\log S$ relation towards higher fluxes. There are indications that the hard sources in NGC 300 follow a similar luminosity function, as is indicated by the different slope for sources with luminosities between $1.5 \times 10^{36} \mathrm{erg} \mathrm{s}^{-1}$ and $\sim 3.5 \times 10^{36} \mathrm{erg} \mathrm{s}^{-1}$, and for sources between $\sim 3.5 \times 10^{36} \mathrm{erg} \mathrm{s}^{-1}$ and $1 \times 10^{37} \mathrm{erg} \mathrm{s}^{-1}$. Due to the low number of sources in our sample, however, constraining the luminosity function in this range is not possible. We also note that the two luminosity functions cross at $\sim 6 \times 10^{36} \mathrm{erg} \mathrm{s}^{-1}$ and that it is the soft sources which are dominating at the highest luminosity levels, as seems to be typical for spiral galaxies (Colbert et al. 2004).

\subsection{The central diffuse emission region}

We extracted the spectrum of the central diffuse emission region after removing all point sources located in that region. The extracted regions are defined by the region task, such that the brightness contour level of the source PSF functions are equal to half of their background flux. We defined the diffuse emission region with a circle of radius $3866^{\prime \prime} 5$, centered on $\alpha_{\mathrm{J} 2000.0}=$ $00^{\mathrm{h}} 54^{\mathrm{m}} 52^{\mathrm{s}} \cdot 4, \quad \delta_{\mathrm{J} 2000.0}=-37^{\circ} 41^{\prime} 07^{\prime \prime} 3$. The background

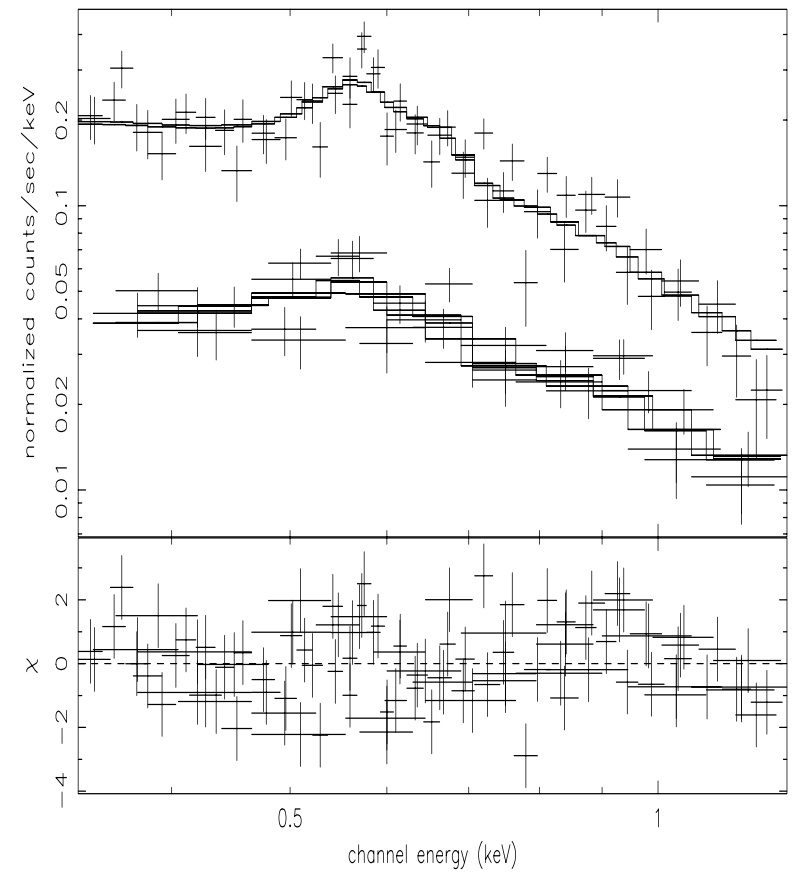

Fig. 6. Top: EPIC pn and MOS spectra of the central diffuse emission region and the best fit spectral model, consisting of the sum of an APEC model, and a bremsstrahlung component, bottom: residuals expressed in $\sigma$.

was taken from an annulus with the same center, an inner radius of 386.'5 and outer radius of 711'. 1 (see Fig. 7).

Data from all instruments and both revolutions were used to extract the spectrum. Because the spectrum of the diffuse emission region is very soft, we consider only the $0.3-1.3 \mathrm{keV}$ energy band. The $\mathrm{Al}$ and $\mathrm{Si}$ fluorescence lines present in the MOS background (in the $1.3-1.9 \mathrm{keV}$ band), which cannot be removed properly, are beyond the region of interest. The spectrum can be described $\left(\chi^{2} /\right.$ d.o.f. $=$ $142.5 / 94)$ by thermal emission from a collisionally ionized plasma, as described by XSPEC's APEC model (see http://hea-www.harvard.edu/APEC/ for a description of this model) with a temperature of $k T=0.2 \pm 0.01 \mathrm{keV}$ plus a thermal component with a temperature of $k T=0.8 \pm 0.1 \mathrm{keV}$. The $0.3-1.3 \mathrm{keV}$ flux is $F_{0.3-1.3}=1.8 \times 10^{-13} \mathrm{erg} \mathrm{cm}^{-2} \mathrm{~s}^{-1}$ (Fig. 6; error bars are at the $90 \%$ level). Similar results are found for the diffuse region in nearly face-on spiral galaxy M 101 (Kuntz et al. 2003), where the spectrum in the $0.5-2 \mathrm{keV}$ band, is characterized by the sum of two thermal spectra with $k T=0.20 \mathrm{keV}$ and $k T=0.75 \mathrm{keV}$.

\section{Properties of the optical counterparts}

To determine the optical counterparts of the X-ray sources we first improved the X-ray aspect solution by comparing the optical and X-ray coordinates of 21 sources inside of the $D_{25}$ disk which have clear optical counterparts. This is done via the eposcorr task, which uses a correlation algorithm to find offsets in RA, Dec, and roll angle which improve the positional accuracy of the X-ray positions with respect to the optical data. These optimum offsets are then used to correct the input source 


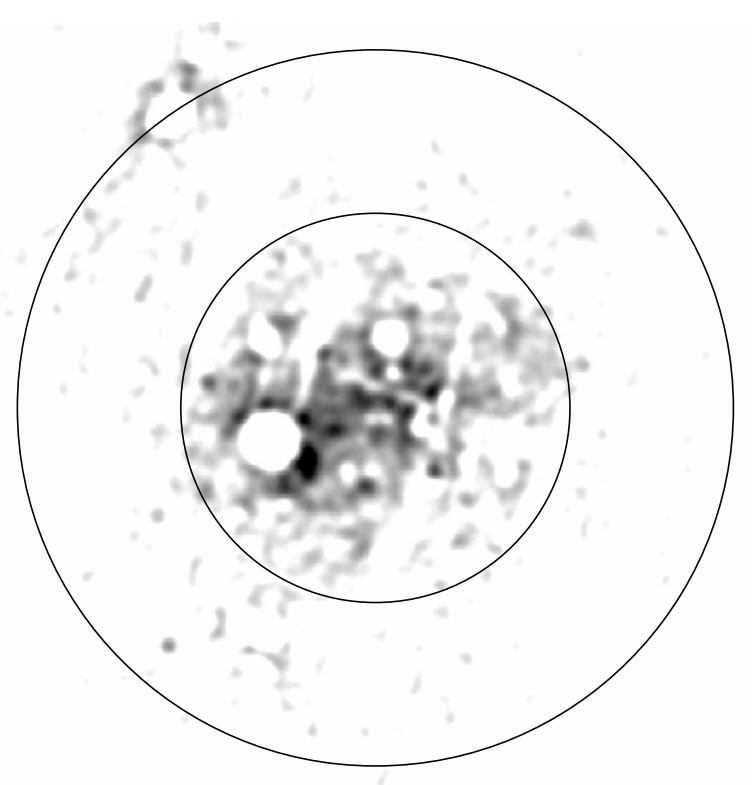

Fig. 7. Smoothed $0.3-1.3 \mathrm{keV} \mathrm{X}$-ray image of the central region of NGC 300 after removal of detected sources. The circle and annulus show the region for the central diffuse emission area and the associated background, respectively.

positions. This algorithm reveals a systematic shift (X-ray optical) of $-1.25^{\prime \prime} \pm 0.31$ in right ascension, of $-0.17^{\prime \prime} \pm 0.31$ in declination, and of $-0.077 \pm 0.27$ for the roll angle.

These offset values are close to values found in the astrometric calibration of XMM-Newton by Guainazzi et al. (2004) ${ }^{1}$, who find $-2.52^{\prime \prime}(1 \sigma)$ and $-3.09^{\prime \prime}(1 \sigma)$ in right ascension for MOS1 and MOS2, and 1.'19 and 0.'41 in declination, respectively. The final uncertainty in X-ray position results from a combination of the edetect_chain output and the error associated with this position offset.

After correcting the X-ray positions, we searched for all possible optical counterparts in the merged $B V R$ optical image and then calculate their fluxes in each of these three optical bands. Photometry was performed with the IDL idlphot photometry library available at http://idlastro.gsfc. nasa.gov/contents.html, which is a set of IDL procedures adapted from an early Fortran version of the DAOPHOT aperture photometry package (Stetson 1987).

We generate an initial optical catalogue by searching for sources within the area surrounding the corrected X-ray positions (for which the radius is given by the uncertainty of the position) in the merged optical image using idlphot's find procedure and assuming a Gaussian point spread function (PSF). This search results in a list of several possible optical counterparts. These source positions are then improved by fitting a measured PSF (as determined from bright optical sources in the image) and the source flux in the $B, V$, and $R$ bands is determined from the PSF fit after subtracting the local background level. Comparing the $B$ and $V$ magnitudes with reference stars given by Pietrzyński et al. (2002b) shows differences of less than $0.15 \mathrm{mag}$, in agreement with our typical flux uncertainty.

\footnotetext{
${ }^{1}$ http://xmm.vilspa.esa.es/external/xmm_sw_cal/ sas_sci_val/index.shtml
}

For the 32 brightest X-ray sources within the $D_{25}$ ellipse, Fig. 8 shows the resulting optical counterparts in the merged optical image. As further described in Sect. 5 below, we also compare these X-ray and optical positions with sources from SIMBAD, the USNO-A2.0 catalog, and with radio sources from Payne et al. (2004). We consider sources from these catalogs as possible counterparts if they have a distance less than $20^{\prime \prime}$ from the corrected X-ray positions for X-ray sources, $10^{\prime \prime}$ for (suspected) supernova remnants and 5 " for the other sources. The closest sources of these counterparts are shown in Fig. 8 with a box.

\section{Summary table}

Table 1 summarizes all information collected from the $86 \mathrm{X}$-ray sources detected inside the $D_{25}$ disk. The first column gives the source ID. The second and third columns give the equatorial sky coordinates of the X-ray sources from the SAS edetect_chain task corrected by the eposcorr task. The combined positional error (in arcsec) from edetect_chain and eposcorr is given in Col. 4. Column 5 lists the detection likelihood and Cols. 6 and 7 give net counts and count rates, respectively (in counts $\mathrm{s}^{-1}$ ), and their corresponding uncertainties. Columns 8 and 9 list the softer and harder hardness ratios defined by Eq. (2) and their errors. Columns 10 and 11 give the $0.3-6.0 \mathrm{keV}$ flux and luminosity, expressed in $\mathrm{erg} \mathrm{cm}^{-2} \mathrm{~s}^{-1}$ and $\mathrm{erg} \mathrm{s}^{-1}$ respectively.

Table 2 summarizes all possible optical counterparts found from the corrected X-ray positions within the X-ray position error circle. Column 1 gives the $\mathrm{X}$-ray source ID and the number in brackets designates a label number when several optical counterparts are found within the X-ray position error area. Columns 2 and 3 give the equatorial sky coordinates of optical counterparts found by idlphot's find procedure. Columns 4-6 give the magnitudes for the optical counterparts, in the $B, V$, and $R$ band respectively, with errors of $\sim 0.15$ mag. Column 7 gives the name and, when available, the reference (within brackets) for possible radio and optical counterparts sources from SIMBAD (which includes the ROSAT sources), the USNO-A2.0, and the following catalogues: Payne et al. (2004, PFP2004), Schild et al. (2003, SCA2003), Read \& Pietsch (2001, RP2001), Pietrzyński et al. (2001, PGF2001), Pannuti et al. (2000, PDL2000), Blair \& Long (1997, BL97), Soffner et al. (1996, SMJ96), Iovino et al. (1996, ICS96), Deharveng et al. (1988, DCL88), Humphreys \& Graham (1986, HG86), Graham (1984, G84).

According to SIMBAD, 14 of our X-ray sources detected inside the optical disk had already been observed in the X-rays (labelled "X"), there are 9 SNR or suspected ones (labelled "SNR?"), 11 radio sources (labelled "radio"), from which three are associated with SNRs and 8 are possible AGNs. Other sources match with association of stars (labelled "Assoc*"), $\mathrm{H}$ II (ionized) regions (labelled "H II"), with regions close to Cepheid variable stars (labelled "Cepheid"), or with stars (labelled "Star"). Many sources also have an USNO-A2.0 optical counterpart (labelled with a number).

As already discussed in Sect. 3.2, our brightest source (\#1), which has a luminosity of $1.70 \times 10^{38} \mathrm{erg} \mathrm{s}^{-1}$, has been 


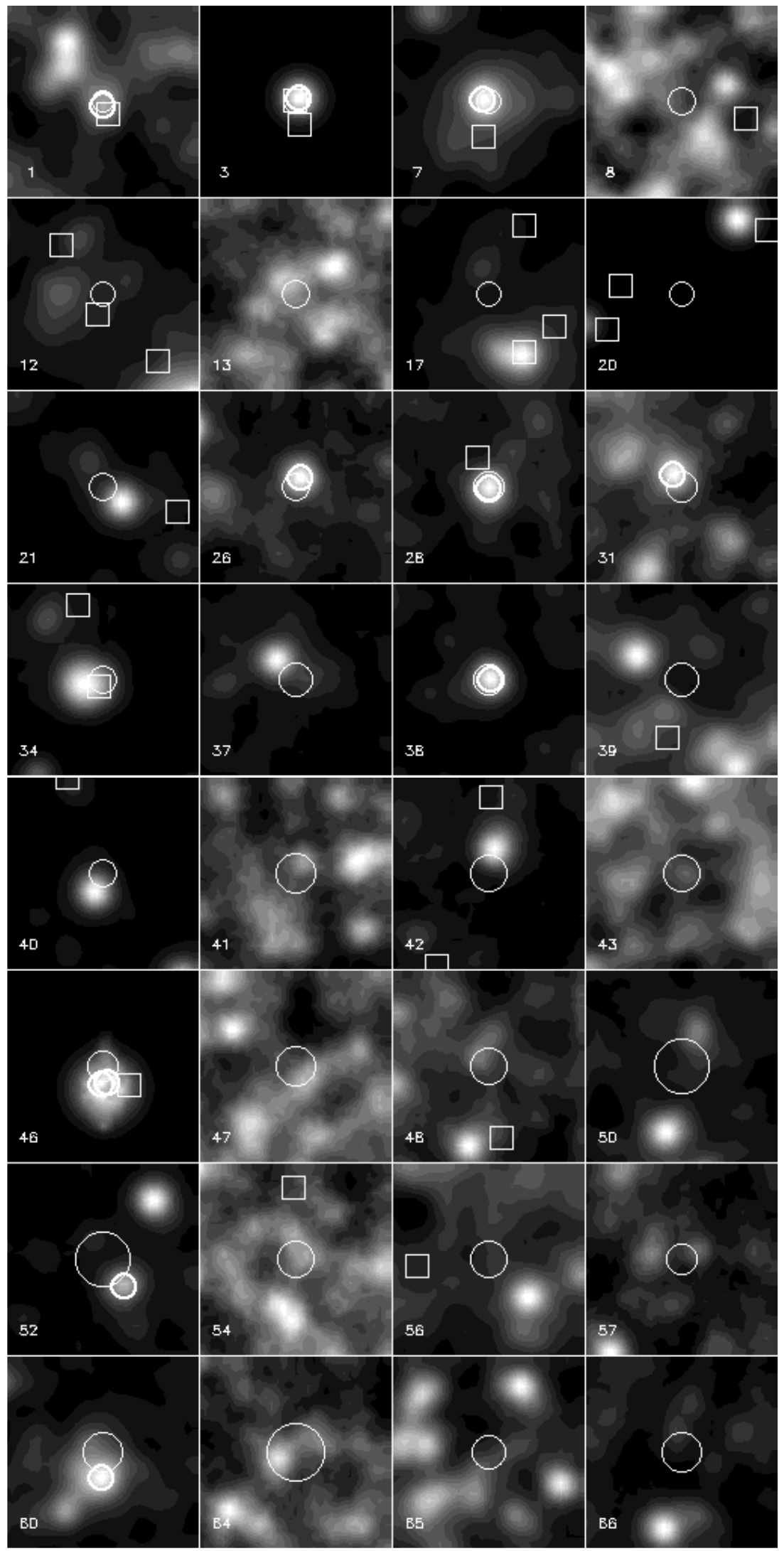

Fig. 8. 7.4" $\times 7.4^{\prime \prime}$ optical images of the region centered on the corrected position of the 32 brightest X-ray sources inside the $D_{25}$ optical disk. The circle indicates the $68 \%$ positional error of the X-ray coordinates, the thicker circles show the optical sources found within the X-ray error circle. Catalog sources having a distance of less than $5^{\prime \prime}$ from the X-ray position are shown with a box. 
Table 2. Summary of the optical counterparts of X-ray sources found in NGC 300 (see text for details).

\begin{tabular}{|c|c|c|c|c|c|c|}
\hline $\begin{array}{l}\text { ID } \\
\text { (1) }\end{array}$ & $\begin{array}{l}\alpha_{\mathrm{J} 2000.0} \\
(2)\end{array}$ & $\begin{array}{l}\delta_{\mathrm{J} 2000.0} \\
(3)\end{array}$ & $\begin{array}{l}\text { mag }(B) \\
(4)\end{array}$ & $\begin{array}{l}\operatorname{mag}(V) \\
(5)\end{array}$ & $\begin{array}{l}\operatorname{mag}(R) \\
(6)\end{array}$ & $\begin{array}{l}\text { comments } \\
\text { (7) }\end{array}$ \\
\hline $1(1)$ & $00: 55: 10.0$ & $-37: 42: 12.2$ & 23.44 & 23.35 & 22.62 & X (RP2001 - P42), WR* (SCA2003 - 41) \\
\hline $3(1)$ & 00:55:10.9 & $-37: 48: 34.2$ & 20.82 & 20.40 & 19.85 & X (RP2001 - P58), radio (PFP2004), USNO: 0450-00323113 \\
\hline $7(1)$ & $00: 54: 50.3$ & $-37: 38: 49.5$ & 22.77 & 21.53 & 20.42 & X (RP2001 - P32), radio (PFP2004) \\
\hline 8 & & & & & & X (XMMU J005510.7-373855), X (RP2001 - P33) \\
\hline 12 & & & & & & SNR (BL97 - N300-S10), HII (SMJ96 - HII W22), HII (BL97 - N300-H10), radio (PFP2004) \\
\hline 13 & & & & & & $\mathrm{X}(\mathrm{RP} 2001-\mathrm{P} 25)$ \\
\hline 17 & & & & & & X (RP2001 - P41), HII (SMJ96 - HII W7), HII (SMJ96 - HII W9), SNR (PDL2000 - SNR 6) \\
\hline 20 & & & & & & SNR (PDL2000 - SNR 15), USNO: 0450-00324001, USNO: 0450-00324043 \\
\hline 21 & & & & & & X (RP2001 - P50) \\
\hline $26(1)$ & $00: 55: 26.2$ & $-37: 38: 37.9$ & 24.33 & 23.69 & 23.07 & X (RP2001 - P30) \\
\hline $28(1)$ & $00: 54: 42.6$ & $-37: 43: 43.2$ & 22.88 & 22.60 & 22.18 & Cepheid (G84 - 14) \\
\hline $31(1)$ & $00: 54: 30.6$ & $-37: 43: 15.4$ & 24.35 & 23.82 & 23.35 & \\
\hline 34 & & & & & & SNR (BL97 - N300-S26), HII (DCL88 - 141), radio (PFP2004) \\
\hline \multicolumn{7}{|r|}{ 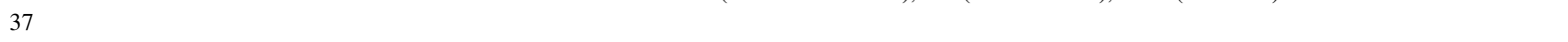 } \\
\hline $38(1)$ & $00: 54: 42.6$ & $-37: 37: 32.7$ & 22.15 & 21.71 & 21.40 & \\
\hline 39 & & & & & & Assoc* (PGF2001 - AS 56a) \\
\hline 40 & & & & & & X (RP2001 - P26) \\
\hline 41 & & & & & & X (RP2001 - P54) \\
\hline 42 & & & & & & Cepheid (G84 - 17), X (RP2001 - P51) \\
\hline \multicolumn{7}{|r|}{ 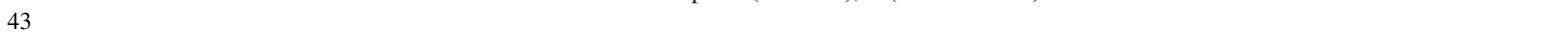 } \\
\hline $46(1)$ & $00: 55: 42.9$ & $-37: 44: 35.5$ & 15.26 & 15.35 & 16.31 & X (RP2001 - P48), USNO: 0450-00326180 \\
\hline $46(2)$ & 00:55:42.9 & $-37: 44: 35.5$ & 15.25 & 15.30 & 16.10 & \\
\hline \multicolumn{7}{|l|}{47} \\
\hline \multicolumn{7}{|l|}{48} \\
\hline \multicolumn{7}{|l|}{50} \\
\hline $52(1)$ & $00: 55: 42.2$ & $-37: 40: 24.7$ & 24.81 & 23.41 & 22.14 & X (RP2001 - P36) \\
\hline 54 & & & & & & radio (PFP2004) \\
\hline 56 & & & & & & HII (SMJ96 - HII C27), radio (PFP2004) \\
\hline \multicolumn{7}{|r|}{ (5) } \\
\hline $60(1)$ & $00: 54: 44.4$ & $-37: 41: 15.8$ & 23.04 & 22.31 & 21.52 & SNR (PDL2000 - SNR 5), X (RP2001 - P41) \\
\hline \multicolumn{7}{|r|}{ 然 } \\
\hline \multicolumn{7}{|l|}{65} \\
\hline 66 & & & & & & X (RP2001 - P50) \\
\hline $67(1)$ & $00: 54: 35.9$ & $-37: 34: 33.9$ & 24.48 & 23.49 & 22.47 & \\
\hline 69 & & & & & & radio (PFP2004) \\
\hline 71 & & & & & & USNO: 0450-00323152 \\
\hline $72(1)$ & $00: 54: 37.6$ & $-37: 42: 49.0$ & 24.98 & 24.35 & 23.62 & SNR (PDL2000 - SNR 3) \\
\hline 73 & & & & & & X (RP2001 - P28) \\
\hline \multicolumn{7}{|l|}{74} \\
\hline $79(1)$ & $00: 54: 22.2$ & $-37: 40: 25.3$ & 21.78 & 21.73 & 21.91 & HII (DCL88 - 10), SNR? (BL97 - N300-S2), USNO: 0450-00318453 \\
\hline \multicolumn{7}{|r|}{$\mathrm{C}^{\circ}$} \\
\hline 87 & & & & & & \\
\hline 88 & & & & & & Star (HG86 - 12), USNO: 0450-00318469 \\
\hline 90 & & & & & & \\
\hline 91 & & & & & & radio (PFP2004) \\
\hline 92 & & & & & & \\
\hline $94(1)$ & $00: 55: 12.2$ & $-37: 38: 23.8$ & 24.00 & 23.58 & 23.11 & \\
\hline 99 & & & & & & $\mathrm{X}(\mathrm{RP} 2001$ - P57) \\
\hline 100 & & & & & & \\
\hline 102 & & & & & & HII (BL97 - N300-H3), Assoc* (PGF2001 - AS 14) \\
\hline 103 & & & & & & \\
\hline 107 & & & & & & USNO: 0450-00321929 \\
\hline 112 & & & & & & \\
\hline $117(1)$ & 00:55:13.9 & $-37: 47: 57.4$ & 24.64 & 24.28 & 23.43 & \\
\hline $120(1)$ & $00: 54: 53.2$ & $-37: 43: 11.3$ & 20.98 & 20.15 & 19.35 & radio (PFP2004) \\
\hline 122 & & & & & & \\
\hline 123 & & & & & & SNR? (BL97 - N300-S19) \\
\hline 125 & & & & & & \\
\hline $126(1)$ & $00: 54: 53.3$ & $-37: 40: 29.0$ & 23.32 & 23.03 & 22.59 & \\
\hline 128 & & & & & & USNO: 0450-00324361 \\
\hline 132 & & & & & & \\
\hline 134 & & & & & & \\
\hline 135 & & & & & & \\
\hline $136(1)$ & $00: 54: 44.8$ & $-37: 37: 40.9$ & 24.16 & 23.81 & 23.53 & \\
\hline 137 & & & & & & \\
\hline $139(1)$ & $00: 55: 7.6$ & $-37: 45: 15.5$ & 24.20 & 24.26 & 24.29 & \\
\hline 140 & & & & & & X (RP2001 - P30) \\
\hline 141 & & & & & & \\
\hline $142(1)$ & $00: 54: 33.2$ & $-37: 44: 4.4$ & 24.80 & 24.33 & 23.99 & \\
\hline 143 & & & & & & \\
\hline
\end{tabular}


Table 2. continued.

\begin{tabular}{|c|c|c|c|c|c|c|}
\hline $\begin{array}{l}\text { ID } \\
(1)\end{array}$ & $\begin{array}{l}\alpha_{\mathrm{J} 2000.0} \\
(2)\end{array}$ & $\begin{array}{l}\delta_{\mathrm{J} 2000.0} \\
(3)\end{array}$ & $\begin{array}{l}\operatorname{mag}(B) \\
(4)\end{array}$ & $\begin{array}{l}\operatorname{mag}(V) \\
(5)\end{array}$ & $\begin{array}{l}\operatorname{mag}(R) \\
(6)\end{array}$ & $\begin{array}{l}\text { comments } \\
(7)\end{array}$ \\
\hline $145(1)$ & $00: 55: 1.0$ & $-37: 34: 40.5$ & 19.87 & 18.50 & 17.22 & USNO: 0450-00322130 \\
\hline \multicolumn{7}{|l|}{146} \\
\hline $147(1)$ & $00: 54: 51.8$ & $-37: 47: 8.6$ & 24.87 & 24.25 & 23.26 & \\
\hline \multicolumn{7}{|l|}{148} \\
\hline \multicolumn{7}{|l|}{150} \\
\hline $151(1)$ & $00: 55: 33.9$ & $-37: 43: 12.8$ & 21.28 & 21.07 & 20.71 & $\begin{array}{l}\text { SNR? (BL97 - N300-S28), HII (S66b - 80), HII (DCL88 - 159), Star (BGK2002 - A14), } \\
\text { radio (PFP2004), USNO: 0450-00325259 }\end{array}$ \\
\hline $151(2)$ & $00: 55: 33.8$ & $-37: 43: 9.3$ & 24.15 & 24.10 & 24.28 & \\
\hline \multicolumn{7}{|l|}{152} \\
\hline \multicolumn{7}{|l|}{153} \\
\hline $155(1)$ & $00: 54: 56.8$ & $-37: 43: 41.6$ & 24.83 & 24.04 & 23.40 & \\
\hline $155(2)$ & $00: 54: 56.9$ & $-37: 43: 38.7$ & 24.27 & 24.14 & 24.44 & \\
\hline $156(1)$ & $00: 54: 30.0$ & $-37: 40: 31.2$ & 24.83 & 23.95 & 23.48 & \\
\hline $157(1)$ & $00: 54: 52.7$ & $-37: 46: 2.1$ & 25.67 & 24.78 & 23.65 & \\
\hline $158(1)$ & $00: 54: 53.4$ & $-37: 44: 40.7$ & 21.66 & 21.56 & 21.54 & USNO: 0450-00321373 \\
\hline $158(2)$ & $00: 54: 53.3$ & $-37: 44: 39.2$ & 23.27 & 22.45 & 21.58 & \\
\hline $159(1)$ & $00: 54: 46.2$ & $-37: 47: 19.0$ & 23.61 & 22.73 & 21.47 & \\
\hline 160 & & & & & & HII (DCL88 - 99), radio (PFP2004) \\
\hline $161(1)$ & $00: 54: 41.5$ & $-37: 43: 3.9$ & 22.89 & 21.24 & 20.07 & \\
\hline $162(1)$ & $00: 54: 46.0$ & $-37: 45: 21.1$ & 23.90 & 22.31 & 21.14 & \\
\hline $163(1)$ & 00:55:11.6 & $-37: 40: 13.8$ & 23.23 & 23.02 & 23.22 & \\
\hline
\end{tabular}

identified by (source P42) Read \& Pietsch (2001), as a possible accreting binary. This source has been found to have a Wolf Rayet star as optical counterpart (labelled "WR*"). The source has a luminosity close to the Eddington limit for a $1.4 M_{\odot}$ compact object, which may suggest the presence of a black hole or neutron star X-ray binary. Source number 8 has already been identified by Read \& Pietsch (2001) and Kong \& Di Stefano (2003) as a luminous supersoft X-ray source and has no optical counterpart.

\section{Conclusions}

In this paper we have described the global properties of the detected X-ray sources in NGC 300 as found in the XMM-Newton data. A total of 163 sources were found using the $0.3-6 \mathrm{keV}$ EPIC data of orbits 192 and 195, of which 86 are located within the optical $D_{25}$ disk. This increases the X-ray inventory of NGC 300 by a factor of $\sim 3$. This increment is mainly due to the better sensitivity of our observations, caused by the higher effective area of XMM-Newton with respect to ROSAT.

Using the color-color diagram allowed us to determine the shape of the X-ray spectrum for each source individually and to estimate the source fluxes. The luminosity function of NGC 300 is similar to that of other spirals (Colbert et al. 2004) and can be described by a power law with slope $1.17 \pm 0.17$. It is dominated by soft sources at high luminosities, although we do not find strong super-Eddington sources in the galaxy. More information about the brightest X-ray sources inside the optical disk, such as spectral fitting and temporal analyses, will be given in a subsequent paper. The spectrum of the central diffuse emission region can be described $\left(\chi_{\text {red }}^{2}=1.52\right)$ by thermal emission from a collisionally ionized plasma with $k T=0.2_{-0.01}^{+0.01} \mathrm{keV}$, plus a second thermal component with a temperature of $k T=0.8_{-0.1}^{+0.1} \mathrm{keV}$.

The SAS eposcorr task revealed a small positional offset. After having corrected for this offset, we searched for optical counterparts in the $B, V$, and $R$ data and cross-correlate with sources from SIMBAD and USNO-A2.0 catalogs, and radio sources.

We identified possible optical and radio counterparts to all X-ray sources using a variety of catalogues. The brightest $\mathrm{X}$-ray source is probably a black hole or neutron star X-ray binary, possibly accreting from a Wolf Rayet star which was identified as the most likely optical counterpart. We confirm the presence of a luminous supersoft X-ray source which has no optical counterpart.

Acknowledgements. We thank Jeffrey Payne for kindly providing his list of radio source coordinates and for his collaboration. We also thank Wolfgang Pietsch for useful discussions and the referee, Andy Read, for his thorough refereeing which greatly improved the quality of this publication.

This paper is based on observations with XMM-Newton, an ESA science mission with instruments and contributions directly financed by the ESA Member States and the USA (NASA), and on observations made with ESO Telescope at La Silla observatory and retrieved from the ESO archive. We acknowledge partial support from DLR grant 500X0002. This work was furthermore supported by the BMBF through the DLR under the project 50OR0106, by the BMBF through DESY under the project 05AE2PDA/8, and by the Deutsche Forschungsgemeinschaft under the project SCHN 342/3-1. The support given by ASTROVIRTEL, a project funded by the European Commission under FP5 Contract No. HPRI-CT-1999-00081, is acknowledged. This research has made use of the SIMBAD database, operated at CDS, Strasbourg, France.

\section{References}

Blair, W. P., \& Long, K. S. 1997, ApJS, 108, 261

Carpano, S., Wilms, J., Schirmer, M., \& Kendziorra, E. 2004, Mem. Soc. Astron. Italiana, 75, 486

Colbert, E. J. M., Heckman, T. M., Ptak, A. F., Strickland, D. K., \& Weaver, K. A. 2004, ApJ, 602, 231

Crawford, D. F., Jauncey, D. L., \& Murdoch, H. S. 1970, ApJ, 162, 405 
de Vaucouleurs, G., de Vaucouleurs, A., Corwin, jr., H., et al. 1991, Third Catalogue of Bright Galaxies (New York: Springer)

Deharveng, L., Caplan, J., Lequeux, J., et al. 1988, A\&AS, 73, 407

Dickey, J. M., \& Lockman, F. J. 1990, ARA\&A, 28, 215

Freedman, W. L., Madore, B. F., Gibson, B. K., et al. 2001, ApJ, 553, 47

Graham, J. A. 1984, AJ, 89, 1332

Grimm, H.-J., Gilfanov, M., \& Sunyaev, R. 2002, A\&A, 391, 923

Guainazzi, M., Gabriel, C., Altieri, B., et al. 2004, XMM-SOC-USRTN-0009, Issue 1.1

Humphrey, P. J., \& Buote, D. A. 2004, ApJ, 612, 848

Humphreys, R. M., \& Graham, J. A. 1986, AJ, 91, 522

Iovino, A., Clowes, R., \& Shaver, P. 1996, A\&AS, 119, 265

Kendziorra, E., Wilms, J., Lamer, G., \& Staubert, R. 2001, in New Visions of the X-ray Universe in the XMM-Newton and Chandra Era, ed. F. Jansen, et al., ESA SP-488, ESA Publications, Noordwijk, in press

Kong, A. K. H., \& Di Stefano, R. 2003, ApJ, 590, L13

Kuntz, K. D., Snowden, S. L., Pence, W. D., \& Mukai, K. 2003, ApJ, 588,264

Nowak, M. A., Wilms, J., Heindl, W. A., et al. 2001, MNRAS, 320, 316
Pannuti, T. G., Duric, N., Lacey, C. K., et al. 2000, ApJ, 544, 780

Payne, J. L., Filipović, M. D., Pannuti, T. G., et al. 2004, A\&A, 425, 443

Pietrzyński, G., Gieren, W., Fouqué, P., \& Pont, F. 2001, A\&A, 371, 497

Pietrzyński, G., Gieren, W., Fouqué, P., \& Pont, F. 2002a, AJ, 123, 789

Pietrzyński, G., Gieren, W., \& Udalski, A. 2002b, PASP, 114, 298

Read, A. M., \& Pietsch, W. 2001, A\&A, 373, 473

Schild, H., Crowther, P. A., Abbott, J. B., \& Schmutz, W. 2003, A\&A, 397,859

Schirmer, M., Erben, T., Schneider, P., et al. 2003, A\&A, 407, 869

Soffner, T., Mendez, R. H., Jacoby, G. H., et al. 1996, A\&A, 306, 9

Stetson, P. B. 1987, PASP, 99, 191

Strüder, L., Briel, U., Dennerl, K., et al. 2001, A\&A, 365, L18

Turner, M. J. L., Abbey, A., Arnaud, M., et al. 2001, A\&A, 365, L27

Ueda, Y., Akiyama, M., Ohta, K., \& Miyaji, T. 2003, ApJ, 598, 886

Williams, B. F., Garcia, M. R., Kong, A. K. H., et al. 2004, ApJ, 609, 735

Wilms, J., Nowak, M. A., Pottschmidt, K., et al. 2001, MNRAS, 320, 327 